University of Nebraska - Lincoln

DigitalCommons@University of Nebraska - Lincoln

1989

\title{
Energetic Electron Tracks and DNA Strand Breaks
}

\author{
A. Chatterjee \\ Lawrence Berkeley National Laboratory \\ W. R. Holley \\ Lawrence Berkeley National Laboratory
}

Follow this and additional works at: https://digitalcommons.unl.edu/usdoepub

Part of the Bioresource and Agricultural Engineering Commons

Chatterjee, A. and Holley, W. R., "Energetic Electron Tracks and DNA Strand Breaks" (1989). US

Department of Energy Publications. 81.

https://digitalcommons.unl.edu/usdoepub/81

This Article is brought to you for free and open access by the U.S. Department of Energy at

DigitalCommons@University of Nebraska - Lincoln. It has been accepted for inclusion in US Department of Energy

Publications by an authorized administrator of DigitalCommons@University of Nebraska - Lincoln. 


\title{
ENERGETIC ELECTRON TRACKS AND DNA STRAND BREAKS
}

\author{
A. Chatterjee and W. R. Holley \\ Division of Cell and Molecular Biology, Lawrence Berkeley Laboratory, University of California, \\ Berkeley, CA 94720, U.S.A.
}

(Received 31 January 1989)

\begin{abstract}
A theoretical model with no adjustable parameters is presented to evaluate the strand break yields for incident electrons between $100 \mathrm{eV}$ and $1 \mathrm{MeV}$. Indirect mechanisms as well as direct mechanisms are included for the production of strand breaks. The model includes the following features: (i) multiple scattering of low energy electrons; (ii) decay of hydroxyl radicals in an aqueous solution containing Tris buffer; (iii) Monte Carlo simulation of the motion of hydroxyl radicals for interaction with the DNA sites; and (iv) stochastic aspects of the direct ionization on the DNA sites and the use of oscillator strength of a DNA molecule. The model is presented using numerical values characteristic of a dilute aqueous solution of SV40 DNA $(10 \mu \mathrm{g} / \mathrm{ml})$ containing $10 \mathrm{mM}$ of Tris. The results have been expressed in terms of yields (indirect and direct) and $\mathrm{D}_{37}$ (indirect only) values as a function of electron energy. The yields have been normalized to breaks/rad/dalton. In the absence of experimental data with different energy electrons, the results of the present calculations have been folded into the estimation of strand breaks induced by heavy charged particles. When these results are compared with experimental data for mammalian cells under conditions such that enzymatic strand break repair is negligible, there is good qualitative agreement with the model. With the expectation that experimental data will soon be available with photons, the present model has been used to predict the strand break yields with electromagnetic radiation for thick as well as thin targets.
\end{abstract}

\section{INTRODUCTION}

IRRESPECTIVE of the quality of a given ionizing radiation, the primary mechanism of energy loss is the interaction of charged particles with the bound electrons of the medium molecules resulting in the production of free electrons. These electrons, sometimes referred to as secondary electrons, are generated over a wide distribution in energy but the physical cross-sections for their production are higher for the lower energy segment of the secondary electron spectrum. If these ejected electrons have enough energy to cause additional ionizations, further electrons (tertiary) are produced. Hence, in radiation-induced damage to a system, the secondary, tertiary, etc., electrons can contribute quite significantly and their effects must be evaluated along with the effects due to the primary radiation.

Unless the electrons are produced adjacent to each other, or there is considerable overlap between their trajectories due to multiple scattering (important for low-energy electrons), a knowledge of the damaging effects of individual electron tracks is fundamental in the estimation of radiation damage. For example, if the yields of DNA strand breaks are to be evaluated due to a given quality of radiation, the contribution from different energy electron tracks should be an important consideration.
Although experimental measurements with monoenergetic X-rays are becoming popular in radiobiology (Goodhead et al., 1979), there are no such studies with monoenergetic low energy electrons due to practical difficulties. Hence, at the present time, theoretical calculations seem to be the only alternative for these electrons. In spite of this fundamental need, almost no theory is available which allows one to estimate radiation effects by considering individual electron tracks. Magee and Chatterjee (1978) have calculated the ferric yields in the Fricke dosimeter system for electron energies ranging between $100 \mathrm{eV}$ and $10 \mathrm{MeV}$. This, perhaps, is the only example of this type of calculation and such studies are needed in the field of radiation biology. For heavy charged particles, theoretical calculations are available where the biological effects of secondary electron tracks have been calculated (Katz et al., 1985). However, in these calculations, the concept of average radial energy density around a heavy charged particle trajectory has been used, instead of individual electron tracks. It may be that these calculations are fairly accurate and simpler for the overall estimation of biological effects due to a given quality of radiation, but an independent approach based on individual electron tracks and a subsequent comparison with the method based on an average energy density approach is necessary in order to have better confidence in the simpler method. 
In this paper, a theoretical calculation is presented for the evaluation of DNA strand break yields for electron energies ranging between $100 \mathrm{eV}$ and $1.0 \mathrm{MeV}$. The complex situation that exists in a cellular system has been avoided by considering an aqueous solution of Simian Virus (SV40) DNA containing $10 \mathrm{mM}$ of Tris buffer. The purpose of Tris is to reduce the number of hydroxyl radicals that can react with the DNA molecule and at this concentration the characteristic diffusion length of $\mathrm{OH}$ is $200 \AA$, which is qualitatively similar to the situation that exists in a cellular environment.

Contributions to the formation of strand breaks from water radicals (indirect mechanism) as well as direct ionization of the sugar ring (direct mechanism) have been considered. In the energy deposition mechanism the effects of multiple scattering of electrons with energies between 100 and $1600 \mathrm{eV}$ have been included.

In the absence of experiments with different energy electrons, no direct comparison of the theoretical results could be made. However, the results of these calculations have been folded into the estimation of effects due to heavy charged particles where secondary electrons play an important role in producing strand breaks. The yields of strand breaks have been normalized/rad/dalton and in this manner the results of the calculation will enable us in the future to compare them with experimental data on strand breaks in a cellular system (with no enzymatic repair) when irradiated with particulate radiation. With the expectation that experimental data on strand breaks will soon be available with photons of different energies, theoretical calculations have been extended for this type of radiation also.

\section{METHOD OF CALCULATION}

The overall procedure has been divided into two parts for calculating the yields of strand breaks. It is well known that both indirect and direct mechanisms are involved in producing this type of damage (Roots et al., 1985). We will now describe these two mechanisms and how they have been incorporated in the calculation.

\subsection{Indirect mechanism}

When double-stranded DNA molecules are in an aqueous medium, radiation interaction with water molecules is a highly probable phenomenon. In this interaction, $\cdot \mathrm{OH}, \cdot \mathrm{H}, \mathrm{e}_{\mathrm{aq}}^{-}$, and $\mathrm{H}_{3} \mathrm{O}^{+}$are the main species that are produced. For the purpose of strand breaks in presence of $\mathrm{O}_{2}, \cdot \mathrm{OH}$ is the only radical that contributes to this type of damage (Hutchinson, 1985 ) by abstracting hydrogen atoms from the five carbon positions on the sugar ring. The rest of the water radicals react with the bases without producing strand breaks.
In order to assess the yield of strand breaks by the indirect mechanism, it is important to consider the following reactions: (i) a given $\mathrm{OH}$ can react with other water radicals (siblings) giving molecular prod. ucts; (ii) a given $\mathrm{OH}$ can be scavenged by Tris producing Tris radicals; and (iii) a given $\cdot \mathrm{OH}$ can diffuse and then attack the various sites (sugars and bases) on a DNA molecule. Obviously, the first two of these reactions do not lead to strand breaks. Hence, for a given electron track, one needs to know the initial position of a given $\mathrm{OH}$ radical in relation to other siblings. Then by simulating the diffusive motion of this radical (Chatterjee and Magee, 1985) one can determine the survival probability in reactions (i) and (ii) and then calculate the probability of reaction with the DNA. The details of this basic idea, applicable for any ionizing radiation, have been published elsewhere (Chatterjee et al., 1986). The simulation of the diffusive motion is accomplished through finite jumps of a given $\mathrm{OH}$ and on each jump the fate of the radical is decided by a Monte Carlo technique which uses random numbers and a decay curve for hydroxyl radicals. This decay curve is constructed on the basis of $\cdot \mathrm{OH}$ reactions with each other and $\cdot \mathrm{H}, \mathrm{e}_{\mathrm{aq}}^{--}, \mathrm{H}_{3} \mathrm{O}^{+}$, and Tris. From this decay curve one can determine the probability of survival of an $\mathrm{OH}$ under observation in each jump time. Based on this probability, whether the radical has survived or not is determined by calling a random number out of uniformly distributed random numbers between 0 and 1 . If the random number is less than the survival probability, the radical is allowed to make another jump and this procedure is followed until either the radical is lost from the system as a molecular product, or it reacts with the DNA molecule. For a given electron track this procedure is repeated about 500,000 times to obtain reproducibility within $\pm 2 \%$.

In order to determine whether a given $\cdot \mathrm{OH}$ has reacted with a base or a sugar molecule, we have considered the structure of the SV40 DNA in a three-dimensional configuration. Assuming the polymer to be in the B form and using the X-ray diffraction data of Arnott and Hukins (1972), such a structure was constructed where each atom on the DNA molecule was placed with its appropriate Cartesian coordinates. Around the sugar ( $\mathrm{C}^{\prime}$ position), a sphere of radius $1 \AA$ was drawn to represent the reactivity of this site with $\mathrm{OH}$. This radius was determined by using the Smoluchowski theory, which relates this reaction radius to the measured rate constant of chemical reaction between $\mathrm{OH}$ and an isolated sugar or base according to the equation

$$
4 \pi r D_{\mathrm{OH}}=k_{\mathrm{OH}-\mathrm{DNA}}
$$

where $r$ is the reaction radius, $D_{\mathrm{OH}}$ is the diffusion constant of $\cdot \mathrm{OH}=2 \times 10^{-5} \mathrm{~cm}^{2} / \mathrm{s}$ and $k$ is the appropriate (sugar or base) rate constant and is known from experimental measurements. Equation (1) assumes that relative movement of a DNA molecule is much smaller than that of a hydroxyl radical. Thus, 
for evaluating the strand break yields due to indirect mechanisms, it is the curve relating the survival probability of a hydroxyl radical with the passage of time, also known as the decay curve, that is the most crucial aspect of the calculation.

Since the strand breaks have been evaluated for different energy electrons, it is necessary to determine the respective decay curves. The concepts of spurs, blobs and short tracks (Mozumder and Magee, 1966) have been useful in obtaining them. In water, energy loss between 6 and $100 \mathrm{eV}$ in a localized region is called a spur, between 100 and $500 \mathrm{eV}$ is called a blob and between 500 and $5000 \mathrm{eV}$ is known as a short track. In order to calculate the decay curves, we have used our previously developed model for the Fricke dosimeter system (Magee and Chatterjee, 1978).

It is well known that low energy electrons undergo multiple scattering and hence their trajectories are such that in all likelihood the spurs created by such tracks overlap. Using the criterion first introduced by Bethe et al. (1938) and taking the average spur size to be $40 \AA$ in diameter, all electrons with energies less than $1600 \mathrm{eV}$ have been treated as one category in which the radicals intermingle with each other in a volume determined by their root mean square penetration depths. Above $1600 \mathrm{eV}$, the electron paths have been taken as linear.

2.1.1. Electron energies between 100 and $1600 \mathrm{eV}$. The essential features of these tracks are such that the initial radical distributions form a single overlapping pattern and as the diffusion continues, the overall track shapes become spherical. This behavior can be represented by the use of an ellipsoid. We chose a prolate spheroid with an initial major axis $b$ given by

$$
b^{2}=\frac{\left\langle r^{2}\right\rangle}{4}-a^{2}
$$

where $\left\langle r^{2}\right\rangle$ is the mean square penetration of an electron and $a$ is the minor axis. For $\cdot \mathrm{H}$ and $\mathrm{e}_{\mathrm{aq}}^{-}$, the values of $a$ are $15 \AA$ and for. $\mathrm{OH}$ and $\mathrm{H}_{3} \mathrm{O}^{+}$, $a=7.5 \AA$. These values were chosen to give reasonable ferric yields. For an aqueous DNA system, these values were not changed.

The prescribed diffusion in prolate spheroid geometry is used to calculate the $\cdot \mathrm{OH}$ decay curve. The number of $\mathrm{OH}$ radicals, $N(\Delta t)$, remaining after time $\Delta t$ is given by the following equations

$$
\begin{aligned}
& 1 / N(\Delta t)-1 / N_{0}=(3 / 4 \pi) \sum_{j=1}^{4} \frac{2 k_{i j}}{4 D_{\mathrm{OH}}\left(b^{2}-a^{2}\right)^{1 / 2}} \\
& \times \ln \frac{\sqrt{b^{2}+4 D_{\mathrm{OH}} \Delta t}-\sqrt{b^{2}-a^{2}}}{\sqrt{b^{2}+4 D_{\mathrm{OH}} \Delta t}+\sqrt{b^{2}-a^{2}} b-\sqrt{b^{2}-a^{2}}} .
\end{aligned}
$$

The summation takes place for four different values of $j$ representing the reactions of a given $\mathrm{OH}$ with (1) $\mathrm{OH}$, (2) $\cdot \mathrm{H}$, (3) $\mathrm{e}_{\mathrm{aq}}^{-}$, and (4) $\mathrm{H}_{3} \mathrm{O}^{+}$. In the above equation, $N_{0}$ is the initial number of $\cdot \mathrm{OH}$ produced and is given by $N_{0}=E / I$, where $E$ is the energy of the electron having a mean square penetration, $\left\langle r^{2}\right\rangle$, and $I=17 \mathrm{eV}$ is the energy required to create either a pair of $(\cdot \mathrm{H}$ and $\cdot \mathrm{OH})$ or $\left(\mathrm{e}_{\mathrm{aq}}^{-}\right.$and $\left.\cdot \mathrm{OH}\right) . \mathrm{H}_{3} \mathrm{O}^{+}$is produced whenever $\mathrm{e}_{\mathrm{aq}}^{-}$is produced to conserve the charge balance. The relative frequencies with which $\mathrm{e}_{\mathrm{aq}}^{-}$and $\cdot \mathrm{H}$ are produced are respectively given by $(E / I)$ $(5.0 / 5.88)$ and $(E / I)(0.88 / 5.88)$ and for $17 \mathrm{eV}$ energy deposition, one $\mathrm{OH}$ is always produced.

From equation (3), one can obtain a decay curve for $\mathrm{OH}$ in pure water. However, since in the DNA system there are Tris molecules which can scavenge . OH very efficiently ( $k=3 \times 10^{9} \mathrm{l} /$ mole s), there is an additional channel for the decay of $\mathrm{OH}$. In order to account for that, the number of surviving $\mathrm{OH}$ as given by equation (3) was further reduced by a factor, $\exp \left(-\Delta t / \tau_{\mathrm{c}}\right)$, where $\tau_{\mathrm{c}}=1 / k C . k$ is the rate constant for $\cdot \mathrm{OH}$ reaction with Tris and $C$ is the concentration of Tris, which is equal to $10 \mathrm{mM}$ in the present solution. In this manner, we have obtained the decay curve for $\mathrm{OH}$ in the presence of Tris and hence at any given instant of time the survival probability of a hydroxyl radical under observation can be obtained.

2.1.2. Electron energies between 1600 and $20 \mathrm{keV}$. Electrons with energies greater than $1600 \mathrm{eV}$ create straight tracks and the radical diffusion is assumed to take place in a cylindrical geometry. These electrons can create separate tracks due to the knock-on collision processes. Their contributions have been accounted for separately using the prescription described in 2.1.1. For the glancing collision processes, spurs are formed and they overlap to create a cylinder provided the energy is less than or equal to $5000 \mathrm{eV}$ giving rise to the formation of short tracks. Above $5000 \mathrm{eV}$, spurs are produced with inter-separation distances which depend upon the mean free path between collisions. For cylindrical geometry, the amount of the radical yield at a time, $\Delta t$, is given by

$$
n(\Delta t)=\sum_{j=1}^{4} \frac{N_{0}}{\left(1+\tau_{i j} / 2\right) \ln \left(1+\Delta t / T^{\prime}\right)} .
$$

In this equation, the initial radical yield per unit path length, $N_{0}$, is $(1-f)(L / I)$ where $f$ is the fraction of energy lost in the knock-on process and $L$ is the stopping power. $\tau_{\mathrm{ij}}$ refers to the ratio of the characteristic times for the diffusion and radical combination processes, $i$ stands for $\cdot \mathrm{OH}$ and $j$ for $\cdot \mathrm{OH}, \cdot \mathrm{H}, \mathrm{e}_{\mathrm{aq}}^{-}$and $\mathrm{H}_{3} \mathrm{O}^{+} . T^{\prime}=\left(a^{2} / 4 D_{\mathrm{OH}}\right)$ is the characteristic time for diffusion and that for radical recombination is $1 / k_{i j} C_{j}$. The decrease in $\mathrm{OH}$ yield is given by equation (4) in pure water and further reduction due to the scavenging action of Tris is accomplished in the same manner as described in 2.1.1. For electron energies greater than $5000 \mathrm{eV}$, the method used to obtain decay curves have been described by Chatterjee et al. (1986).

Thus, the calculations for 2.1.1. give the results for decay of $\mathrm{OH}$ up to $1600 \mathrm{eV}$ and the initial 
calculations of 2.1.2. can extend to $3200 \mathrm{eV}$ because the maximum knock-on electron for this energy is $3200 / 2=1600 \mathrm{eV}$. The next stage can extend to $6400 \mathrm{eV}$, and so on.

Once the respective decay curves are obtained, the Monte Carlo approach described earlier can evaluate the extent to which hydroxyl radicals react with the sugars and the bases. For doublestranded DNA, it has been assumed that only radical attacks on sugars lead to strand breaks. Two strand breaks on opposite strands separated by ten base pairs or less produce a double strand break. From these results, it is simple to calculate the yields of single and double strand breaks for different electron energies.

\subsection{Direct mechanism}

Direct effect calculations for electrons have been separated into two energy regions. Low energy electrons $(<1600 \mathrm{eV})$ have been treated by Monte Carlo techniques using a simple electron track model. The calculations have been extended beyond $1600 \mathrm{eV}$ using an integral equation developed by Magee and Chatterjee (1978). This criterion for energy separation is similar to that described in the section on the indirect mechanism.

We have represented the collective energy deposition paths of a primary and associated secondary electrons as uniformly occupying a cylindrical volume of length $\left(X_{\mathrm{rms}}\right)$ equal to the rms range of the primary electron and radius $\left(r_{\text {eff }}\right)$ roughly that of a typical spur ( $20 \AA)$.

In the Monte Carlo computation, we consider a collection of electron tracks impinging on a randomly oriented segment of double-stranded DNA. The coordinates of the electron track, its lateral extension, and the DNA model are used to determine which sugar-phosphate molecular groups lie within the cylindrical electron track. For example, within the cylinder at an rms residual range $X_{\text {rms }}$, we use a simplified version of the Bragg rule to estimate the average energy deposited on a DNA molecular group:

$$
\Delta E=\left\langle\frac{\mathrm{d} E}{\mathrm{~d} X_{\mathrm{rms}}}\right\rangle \frac{\sum Z_{i}}{2 \pi r_{\mathrm{eff}}^{2} n_{\mathrm{el}}}
$$

where $\left\langle\mathrm{d} E / \mathrm{d} X_{\text {rms }}\right\rangle$ is the effective LET in water for electrons of energy $E$ and rms residual range $X_{\mathrm{rms}}, n_{\mathrm{el}}$ is the electron density of water, $Z_{i}$ is the atomic number of the $i$ th atom and the summation runs over all atoms in the molecular group. Energy is actually deposited on a DNA molecule in a random or stochastic fashion through electronic excitation. Because detailed cross-sections for DNA excitation processes are not known, we represent them by an average process characterized by a mean excitation energy, $\langle E\rangle=29.9 \mathrm{eV}$ [determined from the DNA oscillator strength measurements of Inagaki et al.,
(1974)]. We have assumed that most DNA excitations lead to ionizations.

The average number of excitations (ionizations) due to collision of the electron with the sugarphosphate molecule is $n=\Delta E /\langle E\rangle$ and we make the reasonable assumption that this number follows a Poisson distribution. Thus the respective probabilities of no excitation, one excitation, two excitations, etc., are

$$
\begin{aligned}
& P_{0}=\mathrm{e}^{-n} \\
& P_{1}=n \mathrm{e}^{-n} \\
& P_{2}=\frac{n^{2}}{2} \mathrm{e}^{-n}
\end{aligned}
$$
by

The probability of at least one excitation is given

$$
P_{\geqslant 1}=1-\mathrm{e}^{-n} \text {. }
$$

To understand the nature of the chemical changes due to direct effects, we must know the reaction sequences that follow ionization of the DNA backbone. It is generally believed that the most likely process is deprotonation of the sugar moiety which leads in turn to strand break formation by the same pathway as hydrogen abstraction in the case of $\cdot \mathrm{OH}$ attack by the indirect effect. In these calculations, we have assumed that energy deposited directly on DNA bases is not an important mechanism for strand break formation in a double-stranded DNA.

In our calculations, for each incident electron, we calculate the probability of ionization, $P_{\geqslant 1}$, for every reaction site within the track cylinder. We generate an "event" by choosing a random number, $r_{\text {\# }}$, for each of these sites. If $r_{\#}<P_{\geqslant 1}$ for a particular site, we consider an ionizing reaction to have occurred with the production of a DNA strand break. Breaks occurring close to each other on opposite strands (i.e. within 10 base pairs) lead to double strand breaks. An event is then classified into one of the following categories:

(1) no breaks (NB);

(2) one or more single strand breaks (SSB);

(3) one or more double strand breaks (DSB).

Consider $N$ incident particle tracks yielding $N_{\text {SSB }}$ single strand breaks and $N_{\mathrm{DSB}}$ double strand breaks and depositing total energy $E_{\mathrm{T}}$ in $\mathrm{eV}$ on the DNA (including bases). The strand break $G$-values in standard units of breaks per $100 \mathrm{eV}$ deposited are given by

$$
\begin{aligned}
G_{\mathrm{SSB}} & =\frac{N_{\mathrm{SSB}}}{E_{\mathrm{T}}} \times 100 \\
G_{\mathrm{DSB}} & =\frac{N_{\mathrm{DSB}}}{E_{\mathrm{T}}} \times 100
\end{aligned}
$$

To extend the calculations beyond $1600 \mathrm{eV}$, have made use of the following integral equation. developed by Magee and Chatterjee (1978): 
$G^{\prime}(E)=0.6 G_{\text {core }}^{\prime}+0.4 \frac{\int_{E_{0}}^{E / 2} G\left(E^{\prime}\right) w\left(E, E^{\prime}\right) \mathrm{d} E^{\prime}}{\int_{E_{0}}^{E / 2} w\left(E, E^{\prime}\right) \mathrm{d} E^{\prime}}$.

Here $G^{\prime}(E)$, the differential $G$-value at energy $E$, is given by a differential core contribution, $G_{\text {core }}^{\prime}$, which we calculate using a Monte Carlo scheme analogous to that for low energy electrons, and an integral over the total $G$-value up to energy $E / 2$ weighted by the spectral density $\left[w\left(E, E^{\prime}\right)\right]$ of knock-on electrons. $E_{0}$ is $100 \mathrm{eV}$ and conventionally separates core interactions $(<100 \mathrm{eV})$ and knock-on interactions. Using this equation, one can step up in energy by a factor of 2 for each iteration. First $G^{\prime}$ is extended from $E / 2$ to $E$; then a second integration, as described by Magee and Chatterjee (1978) yields values of $G$ up to energy $E$.

\section{RESULTS}

The results of the calculations based on the indirect mechanism are represented in Figs 1 and 2. In Fig. 1, the yields of single strand breaks (SSB) and double strand breaks have been plotted as a function of electron energy expressed in $\mathrm{keV}$. The respective yields are normalized to breaks/ $\mathrm{rad} /$ dalton. Over the span of electron energies between $100 \mathrm{eV}$ and $10^{3} \mathrm{keV}$, both of these breaks go through a wide minimum. At lower electron energies (less than $600 \mathrm{eV}$ ), as the energy decreases, fewer and fewer water radicals are produced within spurs or blobs and hence the relative importance of sibling reactions (reactions between water radicals) becomes less. This results in higher yields of single and double strand breaks. With increases in electron energy above about $600 \mathrm{eV}$, more and more spurs are produced, but, because of the reduction in the stopping power values, the inter-separation distance between the spurs becomes progressively larger. Thus the water radical species have less and less chance to interact

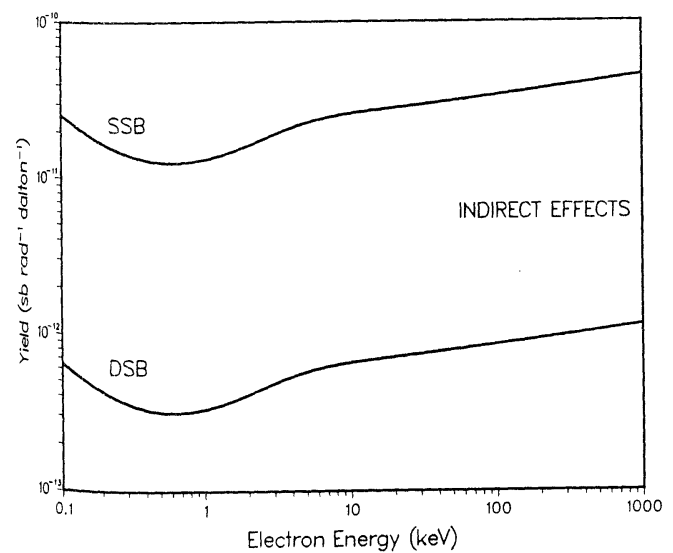

Fig. 1. Yields of strand breaks (contributed by the indirect mechanism only) have been plotted against electron energies. The yields of double strand breaks are about $2-3 \%$ of the single strand breaks.

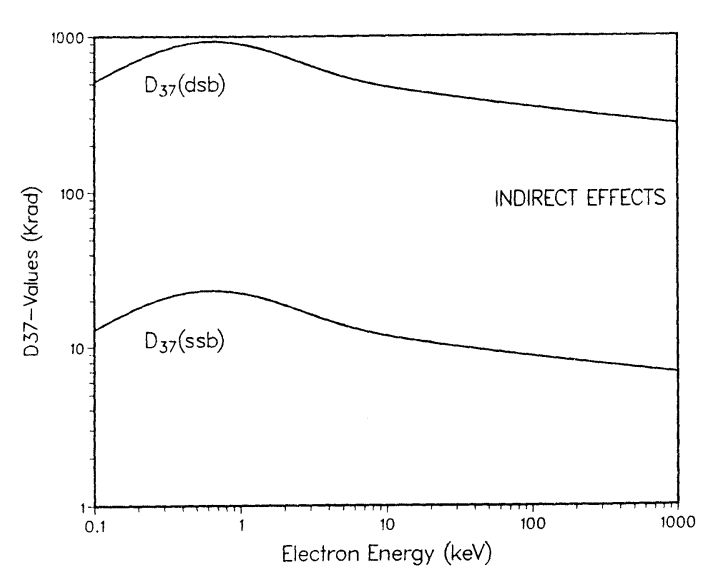

FIG. 2. $D_{37}$ values for single and double strand breaks (for indirect mechanism only) have been plotted against electron energies. Since $D_{37}$ values and yields have a reciprocal relationship, the shapes of these curves are inverse to those of yields as seen from Fig. 1.

with each other from adjacent track entities and hence the yields of strand breaks show a steady increase with the electron energy.

In many experiments, the efficiencies of strand break yields are measured through a determination of $D_{37}$ values, i.e. the dose required to reduce the number of undamaged DNA to $37 \%$ of its initial value. Figure 2 shows a plot of these values as a function of electron energy. $D_{37}(\mathrm{SSB})$ means the dose at which on an average there is a single strand break in each of the DNA molecules present. Similarly, $D_{37}(\mathrm{DSB})$ relates to the corresponding dose for a double strand break. As a function of electron energy, each of these curves goes through a maximum, as expected from the plot of the yield curves (Fig. 1), since yields and $D_{37}$ values have a reciprocal relationship.

The results of calculations based on the direct mechanism for the production of single and double strand breaks by electrons in the energy range $100 \mathrm{eV}-1 \mathrm{MeV}$ have been plotted in Fig. 3. For

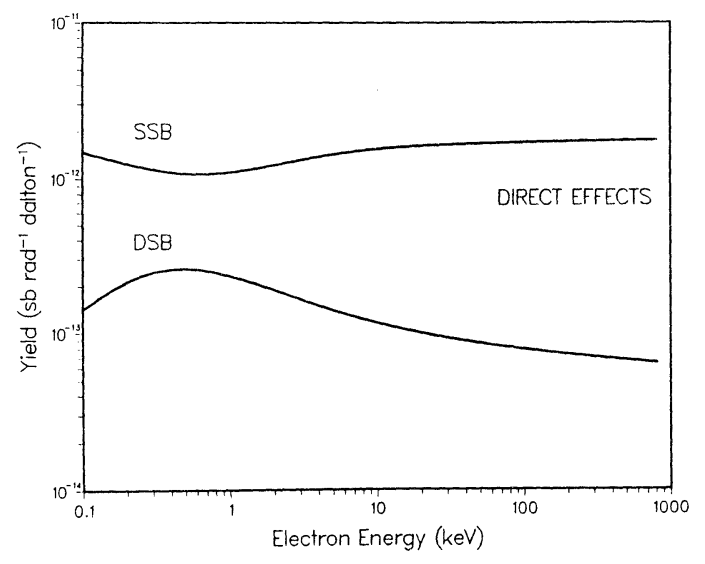

FIG. 3. Calculated strand break yields due to the direct deposition of energy on a DNA molecule are plotted vs incident electron energy. The double strand break yields are about $10 \%$ (less at higher electron energies) of the single strand break yields. 
consistency, we have plotted the yields normalized to breaks/rad/dalton by multiplying the $G$-values by $1.037 \times 10^{-12}$ in order to account for various constants in the calculation.

Some aspects of the behavior of the yields can be understood in the following manner. First, let us consider the double strand break production. At the lowest energies $(\sim 100 \mathrm{eV})$ the tracks are short and total energy available is small, and the probability of hitting both strands and producing independent strand breaks leading to a double strand break is small. The yield increases as more energy is available for deposition on the DNA until the track length becomes longer than the typical distance separating strand breaks on opposite strands which would lead to a double strand break. This separation has been taken to be about 10 base pairs or $30-40 \AA$. As the energy increases, the LET goes down and the energy available for deposition within this "critical" length also decreases. Consequently, the yield of double strand breaks decreases. The single strand break curve is, to a good approximation, a simple reflection of the double strand break curve. In a very simple picture, all energy deposited in the DNA backbone leads to strand breaks (i.e. $\sim 1$ strand break for every $30 \mathrm{eV}$ deposited). Single strand break production should be very roughly independent of electron energy except for the fact that sometimes two (or more) strand breaks become a double strand break and do not count as single strand breaks.

We have used the direct effect electron yields described above in a model for photon interactions to calculate the yields of DNA strand breaks in an aqueous medium. A Monte Carlo computer calculation has been developed in which incident photons of energy $E$ have been allowed to interact in a water target either through Compton scattering or the photoelectric effect (depending on relative

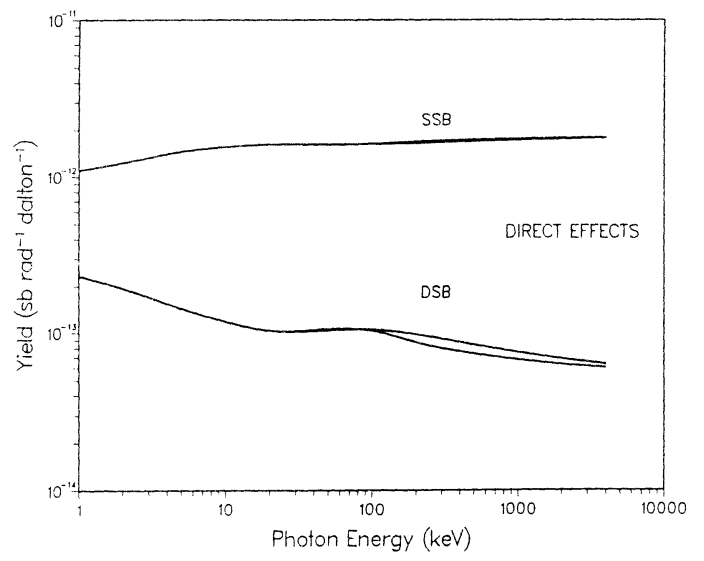

FIG. 4. Theoretical yields of single and double strand breaks due to direct deposition of energy on DNA molecules are plotted vs incident photon energy. The calculation has been made for a totally absorbing "thick" target and a $1 \mathrm{~cm}$ "thin" target. Only for energies above $100 \mathrm{keV}$ are the "thick" target double strand break yields significantly greater than the "thin" target yields. cross-sections). The photons have been followed through the target thickness (an input parameter) adding up contributions of the secondary electrons until the photon is either completely absorbed via a photoelectric interaction or escapes the target. $R_{\boldsymbol{Q}}$. sults of this calculation are plotted for a totally absorbing "thick" target and for a 1-cm "thin" target in Fig. 4. In general, the double strand break yields decrease with increasing photon energy as the average secondary electron energy increases. Note that we have only considered initial photon energies above $1 \mathrm{keV}$ to avoid binding energy complications in the calculation. Essentially all electrons produced by these photon interactions are above $1 \mathrm{keV}$ and in the region where the yield decreases monotonically with increasing electron energy. Only at high energies $(>100 \mathrm{keV})$ are the "thick" target double strand break yields significantly larger than the "thin" target yields. Of course, the single strand break yields are a reflection of the double strand break yields, and vice versa.

\section{DISCUSSION}

One of the ultimate aims of this work is to be able to compare our calculations with strand break data when cells are irradiated with various qualities of radiation. However, in order to do so, the effects of the indirect mechanism have to be recalculated under much higher concentrations of Tris than considered in the present analysis. As mentioned earlier, the concentration of Tris in the system reported here is $10 \mathrm{mM}$ (see Introduction) which translates into an average $\cdot \mathrm{OH}$ migration distance of $200 \AA$. In contrast, the migration distance of an $\mathrm{OH}$ in a cell is only about $30 \AA$. For simulating such a distance in an aqueous solution of DNA, the required concentration of Tris is $500 \mathrm{mM}$. In spite of this requirement, the results of the present calculations can be compared with the experimental measurement of strand breaks in cells irradiated with high LET particles. When the LET is high, the contribution to strand breaks from the indirect mechanism is less than $20 \%$ (Roots et al., 1985). In Fig. 5, such a comparison has been made by using data from reported measurements of several investigators. In these measurements, the experimental conditions were manipulated so that no (or minimum) enzymatic repair of strand breaks was allowed. It can be seen from the comparison that the results are qualitatively similar, providing some confidence in the calculation procedure described in this paper. Even the quantitative agreement at high LET values seems quite reasonable.

It should be pointed out that in the present theoretical model for calculating strand break yields as a function of electron energy, there are no adjustable parameters, and basic constants such as diffusion coefficients, rate of reactions, dimensions of DNA, etc., have entered into the computation as inputs. 
Yield of DNA Strand Breaks

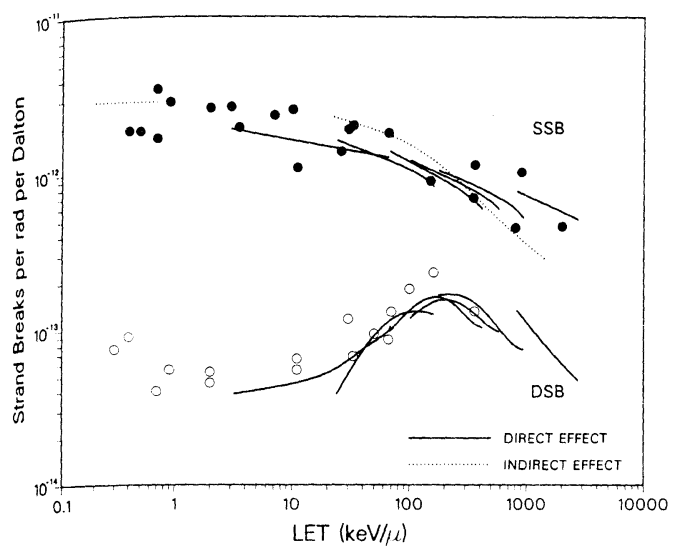

Fig. 5. The calculated yields of single and double strand breaks $/ \mathrm{rad} /$ dalton are plotted vs LET in $\mathrm{keV} / \mu \mathrm{m}$. Solid curves are for direct effects only. Dotted curves are estimated indirect effect yields under conditions which approximately simulate oxygenated cellular systems. For comparison, a selection of experimental measurements from the literature of radiation-induced initial single (O) and double $(O)$ strand break yields using a variety of mammalian cell types is also plotted.

This aspect is quite different from existing models and demonstrates the fact that one can relate the physics of energy deposition with the formation of strand breaks by considering the intermediate chemical phase in the overall evolution of processes of initial (no repair) DNA damage.

In order to make further improvements in the model presented here, several limitations have to be addressed. It is well known that DNA is associated with bound and structured water molecules. What role these molecules play in the ensuing damage process is not clear at the moment and hence has not been accounted for. Similar limitations also exist with respect to migration of deposited energy along a DNA chain. In the present calculation, the deposited energy has been fixed at the given site of interaction and this may not be strictly correct. Other limitations include the neglect of DNA motion, DNA breathing, and the effects of single strand breaks on the DNA structure locally.

In spite of the limitations described above, it is concluded from the present work that the gross features associated with the formation of strand breaks have been taken into account. It is expected that the model may serve as a basic framework for future considerations, both from the point of view of further improvements as well as the calculation of those biological end points which depend on the formation of strand breaks. However, before any more progress can be made with respect to theoretical calculations, experimental data on the yields of strand breaks are urgently needed with low and high energy incident electrons.

Acknowledgement-This work was supported by the Office of Health and Environmental Research, Office of Energy Research, U.S. Department of Energy, under Contract DE-AC03-76SF00098.

\section{REFERENCES}

Arnott S. and Hukins D. W. L. (1972) Optimized parameters for A-DNA and B-DNA. Biophys. Res. Commun. 47, 1504-1509.

Bethe H. A., Rose M. E. and Smith L. P. (1938) Plural and multiple scattering of energetic electrons. Proc. Am. Philos. Soc. 78, 537-569.

Chatterjee A. and Magee J. L. (1985) Theoretical investigation of the production of strand breaks in DNA by water radicals. Radiat. Prot. Dosimetry 13, 137-140.

Chatterjee A., Koehl P. and Magee J. L. (1986) Theoretical consideration of the chemical pathways for radiationinduced strand breaks. Adv. Space Res. 6, 252-270.

Goodhead D. T., Thacker J. and Cox R. (1979) Effectiveness of $0.3 \mathrm{keV}$ carbon ultrasoft $\mathrm{X}$-rays for the inactivation and mutation of cultured mammalian cell. Int. J. Radiat. Biol. 36, 101-114.

Hutchinson F. (1985) Chemical changes induced in DNA by ionizing radiation. Progress. Nucleic Acid Res. 32, $115-153$.

Inagaki T., Hamm R. N., Arakawa E. T. and Painter L. R. (1974) Optical and dielectric properties of DNA in the extreme ultraviolet. J. Chem. Phys. 61, 4246-4250.

Katz R., Dunn D. E. and Sinclair G. L. (1985) Thindown in radiobiology. Radiat. Prot. Dosimetry 13, 281-284.

Magee J. L. and Chatterjee A. (1978) Theory of the chemical effects of high-energy electrons. J. phys. Chem. 82, 2219-2226.

Mozumder A. and Magee J. L. (1966) Theory of radiation chemistry. VII. Structure and reactions in low LET tracks. J. Chem. Phys. 45, 3332-3341.

Roots R., Chatterjee A., Chang P., Lommel L. and Blakely E. A. (1985) Characterization of hydroxyl radicalinduced damage after sparsely and densely ionizing irradiation. Int. J. Radiat. Biol. 47, 157-166. 